\title{
Nutritional services in Northern region of England: comparing with the data obtained in 2003
}

\author{
S. Kiani, B. Davidson and N. P. Thompson on behalf of the Northern Nutrition Network \\ Freeman Hospital, Newcastle University upon Tyne, Newcastle upon Tyne NE7 7DN, UK
}

\begin{abstract}
Malnutrition is commonly found in hospitals among patients and has been shown to be an independent reversible factor. Therefore, provision of a Nutrition Support Service was recommended by The Kings Fund (1992), the British Association for Parenteral and Enteral Nutrition (BAPEN; 1999), Royal College of Physicians (RCP; 2002) and the National Institute of Health and Clinical Excellence (NICE; 2006) as being fundamental to good medical practice. The Northern Nutrition Network was established in 2003 to improve nutrition services across the region.

In 2007, on behalf of this network, we performed an audit to investigate nutrition services in hospitals across the region and compared the data with the similar one performed in 2003. Questionnaires were sent to the sixteen hospitals and results were obtained from all of them (response rate 100\%). In 2003, fourteen hospitals were audited and results were obtained from thirteen of them.

In 2007 only five out of sixteen hospitals had a nutrition support team, of which $60 \%$ had a full-time nutritional nurse specialist, mainly funded through the surgery department. The provision of this service was not significantly different since $2003(P=0.62)$. However, fifteen out of sixteen hospitals now had nutritional steering committees as compared with four hospitals who had this service in $2003(P<0.001)$. Eight hospitals provided a pharmacy manufacturing unit in 2007, which was not significantly different from 2003 ( $P=0.53)$. Three out of sixteen hospitals in 2007 had patients on home Total Parenteral Nutrition (two with only a single patient). Currently, most hospitals (thirteen out of sixteen) were screening the majority of their inpatients on admission for malnutrition (half using Malnutrition Universal Screening Tool). In comparison, only one hospital was screening the majority of out-patients for malnutrition on their first visit. These findings are unchanged since 2003. Only seven out of sixteen hospitals in 2007 had a dedicated service for obese patients with two having a bariatric surgical service.

Since 2003 the number of hospitals with a nutrition steering committee has increased but less than one third of hospitals have a nutrition team or a nutrition nurse specialist, despite advice from NICE. The majority of hospitals screen in-patients but not out-patients for malnutrition.
\end{abstract}

